\title{
MBMUDs: A combinatorial extension of BIBDs showing good optimality behaviour
}

\author{
Pau Bofill \\ Departament d'Arquitectura de Computadors (UPC), Campus Nord-Mòdul-D6, \\ c/ Gran Capità s/n,08071 Barcelona,pau@ac.upc.es \\ Carme Torras \\ Institut de Robòtica i Informàtica Industrial (CSIC-UPC), \\ Parc Tecnològic de Barcelona - Edifici U, \\ c/ Llorens i Artigas 4-6,08028 Barcelona, ctorras@iri.upc.es
}

\begin{abstract}
The construction of a Balanced Incomplete Block Design (BIBD) is formulated in terms of combinatorial optimization by defining a cost function that reaches its lower bound on all and only those configurations corresponding to a BIBD. This cost function is a linear combination of distribution measures for each of the properties of a block design (number of plots, uniformity of rows, uniformity of columns, and balance). The approach generalizes naturally to a super-class including BIBDs, which we call Maximally Balanced Maximally Uniform Designs (MBMUDs), that allow two consecutive values for their design parameters $[r, r+1 ; k, k+1 ; \lambda, \lambda+1]$. In terms of combinatorial balance, MBMUDs are the closest possible approximation to BIBDs for all experimental settings where no set of admissible parameters exists. Thus, other design classes previously proposed with the same approximation aim -such as RDGs, SRDGs and NBIBDs of type I- can be viewed as particular cases of MBMUDs. Interestingly, experimental results show that the proposed combinatorial cost function has a monotonic relation with A- and D- statistical optimality in the space of designs with uniform rows and columns, while its computational cost is much lower.
\end{abstract}

Keywords: block design, maximally balanced design, distribution measure, cost function

\section{Introduction}

Block designs are used in experimental planning with the purpose of maximising the information extracted from a given number of experiments. 
In statistical terms, Balanced Incomplete Block Designs (BIBDs) are know to be optimal according to the usual A, D, and E criteria [16], as well as universally optimal [7], under some simple mathematical models applied to analyse the data, such as the linear model without block effects and the twoway additive model with fixed block effects, although not so for the more elaborate mixed effects model with variable blocksize [9].

Even restraining ourselves to the two simpler models mentioned, BIBDs are of little use in most experimental situations. This is because the parameters of a particular situation often do not satisfy the admissibility conditions of a BIBD. Therefore, a method for constructing efficient block designs for arbitrary parameter values is needed in practice.

Let us be more specific. Consider an experimental setting with $v$ varieties or treatments, $b$ blocks and $u$ experimental units or plots. The admissibility conditions of a BIBD restrict the range of allowed parameter sets to those yielding integer values for the design descriptors: $r=\frac{u}{v}, k=\frac{u}{b}, \lambda=\frac{u(u-b)}{b v(v-1)}$. Several extensions of BIBDs have been proposed for the cases where one or two of these conditions do not hold. Regular Graph Designs (RGDs) $[6,17]$ require strictly uniform rows $(r)$ and columns $(k)$, and allow two consecutive concurrence values $(\lambda, \lambda+1)$. Semi-Regular Graph Designs (SRGDs) [5] and Nearly Balanced Incomplete Block Designs (NBIBDs) of Type I [3] extend this idea to the unequally replicated case by allowing also two consecutive replication values $(r, r+1)$.

In this paper we go one step further and consider the cases where neither of the three admissibility conditions hold. Thus, we define Maximally Balanced Maximally Uniform Designs (MBMUDs), which permit also two consecutive block sizes $(k, k+1)$.

The idea underlying all these extensions is to choose designs that are as balanced as possible. Therefore, they can be generated by minimizing a combinatorial cost function that penalizes unbalance. We begin by defining a family of cost functions whose global minima coincide with BIBDs in the case of admissible parameters (Section 2). The structure of such functions permits deriving the optimal cost analytically, which provides an a priori bound for the minimization. When these functions are applied to arbitrary $(v, b, u)$, the configurations satisfying the derived combinatorial optimal cost, when existing, take at most two consecutive values for each descriptor $[r, r+1 ; k, k+1 ; \lambda, \lambda+1]$, i.e., they are precisely MBMUDs. This is proven in Section 3, where some properties and existence conditions for MBMUDs are also derived.

The statistical interest of these combinatorial extensions of BIBDs is addressed in Section 4. Through experimentation, we find that the proposed combinatorial cost function has a monotonic relation with A- and D-optimality, while its 
computational cost is much lower. Thus, it can be used directly as an approximation of A- and D- efficiencies, or either serve to initialize any algorithm based on a statistical optimality criterion, in order to speed up the minimisation. Another side benefit resulting from the computer implementation is that, for parameter settings for which MBMUDs do not exist but NBIBDs of Type II do exist [3], the proposed procedure converges to this well-established class.

Only binary designs are addressed in this paper, although the work in [14] reveals large classes $(v, b, k)$ where binary designs cannot be optimal with respect to numerous statistical criteria. However, there is no known example of a non binary design that is optimal for A or D. Thus, in focusing on A and $\mathrm{D}$ only we expect to be safe with the binarity restriction.

\section{Combinatorial cost functions for the generation of BIBDs}

Let $\mathcal{A}-v \times b$ be the set of all binary matrices $A \equiv\left[x_{i j}\right]$ with $v$ rows and $b$ columns. Let

$$
\begin{aligned}
o & =\sum_{i=1}^{v} \sum_{j=1}^{b} x_{i j}, \\
r_{i} & =\sum_{j=1}^{b} x_{i j}, \\
k_{j} & =\sum_{i=1}^{v} x_{i j}, \quad \text { and } \\
\lambda_{i k} & =\sum_{j=1}^{b} x_{i j} x_{k j} .
\end{aligned}
$$

Definition 1 (BIBD) For fixed $r, k$ and $\lambda$, with $k<v$ and $\lambda>0$, we say that $A$ is the incidence matrix of a BIBD with parameters $(v, b, u)$ and descriptors $[r, k, \lambda]$ if and only if the following properties are fulfilled:

i) Right number of ones: $o=u^{1}$.

ii) Strictly uniform rows: $r_{i}=r, \quad i=1, \ldots, v$.

iii) Strictly uniform columns: $k_{j}=k, \quad j=1, \ldots, b$.

iv) Strict balance: $\lambda_{i k}=\lambda, \quad i=1, \ldots, v-1, k=i+1, \ldots, v$.

\footnotetext{
${ }^{1}$ Although it can easily be shown that statistically optimal designs must have $o=u$, the variable $o$ is introduced here to allow for the evaluation of matrices with any number of ones, as required in our combinatorial optimization framework.
} 
Parameters and descriptors are related by the following admissibility conditions,

$$
\begin{aligned}
r & =u / v \\
k & =u / b \\
\lambda & =\frac{r(k-1)}{v-1}=\frac{u(u-b)}{b v(v-1)}
\end{aligned}
$$

where $r, k$ and $\lambda$ must be integers. Therefore the range of admissible parameter sets is restricted. Equations (5) and (6) are trivial and condition 1-i is redundant, but it is explicitly defined for presentation purposes. Condition 1-ii can also be derived from 1-iii and 1-iv.

Notice that BIBDs are defined in terms of the uniform distribution of ones over the incidence matrix (see Definition 3 below).

Definition 2 (Cost functions for BIBDs) Given a set $(v, b, u)$ of admissible parameters, we say that $F: \mathcal{A} \rightarrow \mathcal{R}$ is a cost function for BIBD generation if there exists a lower bound $F^{*}$ such that $F\left(A^{*}\right)=F^{*}$ if and only if there exists a $(v, b, u)$-BIBD with incidence matrix $A^{*}$.

Observe that $F^{*}$ may be calculated even if such a design does not exist. For all $A$ corresponding to a non-BIBD configuration, then, it must hold that $F(A)>F^{*}$.

\subsection{Combinatorial distribution measures for the properties of a BIBD}

To illustrate the notion of distribution measure, let us consider the set $\mathcal{B}_{w}$ of all possible arrangements of $w$ balls in $p$ bins, and let $b_{j}$ be the number of balls in bin $j$ in a particular configuration $B \in \mathcal{B}_{w}$, with $w=\sum_{j} b_{j}$.

Definition 3 (Maximal or uniform distribution) We say that $w$ balls are maximally or uniformly distributed over $p$ bins (we say that the number of balls per bin is maximally uniform) if, for a fixed $d$,

$$
b_{j}=d \quad \text { or } b_{j}=d+1, \quad j=1, \ldots, p .
$$

In fact $d=\lfloor w / p\rfloor$, and there will be exactly $w \bmod p$ bins with $d+1$ balls, and the remaining bins will have $d$ balls. In particular, for $(p, w)$ such that $p \mid w$ (multiplicity condition), it holds that $w \bmod p=0$ and all bins will have exactly $d$ balls. We say, in this case, that the distribution is strictly uniform. 
Let us now consider the number of pairs that can be formed by taking balls belonging to the same bin in a given configuration $B \in \mathcal{B}_{w}$,

$$
P(B)=\sum_{j}\left(\begin{array}{c}
b_{j} \\
2
\end{array}\right) .
$$

This measure is quadratic on the $b_{j}$ 's and, therefore, it is minimized for all and only those configurations that are uniformly distributed. We say that $P(B)$ is a measure of the distribution of $w$ balls over $p$ bins.

Extending the measure to any number of balls (i.e., for fixed $p$ but arbitrary $w)$, the lower bound on $P(B)$ may be written as a function of the number of balls $w$,

$$
P^{\min }(w)=p\left(\begin{array}{c}
\lfloor w / p\rfloor \\
2
\end{array}\right)+(w \bmod p)\lfloor w / p\rfloor
$$

and the increment of this lower bound is

$$
\Delta P^{\min }(w)=P^{\min }(w+1)-P^{\min }(w)=\lfloor w / p\rfloor .
$$

Notice that $\Delta P^{\min }(w)$ is a staircase-like function of $w$ with a unit step at each multiple of $p$, and flat regions in between. Therefore, $P^{\min }(w)$ is a piece-wise linear non-decreasing convex function.

Going back to the case of block designs, and over the set $\mathcal{A}-v \times b$ of binary configurations, let us define the following combinatorial measures:

\section{Number of ones:}

$$
U(A)=o=\sum_{i=1}^{v} \sum_{j=1}^{b} x_{i j}
$$

Total pairs of ones:

$$
P_{t}(A)=\left(\begin{array}{l}
o \\
2
\end{array}\right)=\sum_{i=1}^{v} \sum_{k=i}^{v} \sum_{j=1}^{b} \sum_{l=j}^{b} x_{i j} x_{k l}-U(A)
$$

Horizontal pairs of ones:

$$
P_{h}(A)=\sum_{i}\left(\begin{array}{c}
r_{i} \\
2
\end{array}\right)=\sum_{i=1}^{v} \sum_{j=1}^{b-1} \sum_{l=j+1}^{b} x_{i j} x_{i l}
$$

Vertical pairs of ones:

$$
P_{v}(A)=\sum_{j}\left(\begin{array}{c}
k_{j} \\
2
\end{array}\right)=\sum_{j=1}^{b} \sum_{i=1}^{v-1} \sum_{k=i+1}^{v} x_{i j} x_{k j}
$$

\section{Quadruples of ones:}




$$
Q(A)=\sum_{i=1}^{v-1} \sum_{k=i+1}^{v}\left(\begin{array}{c}
\lambda_{i k} \\
2
\end{array}\right)=\sum_{i=1}^{v-1} \sum_{k=i+1}^{v} \sum_{j=1}^{b-1} \sum_{l=j+1}^{b} x_{i j} x_{i l} x_{k j} x_{k l}
$$

The measures $U(A)$ and $P_{t}(A)$ are linear and quadratic, respectively, with the number of ones in $A$ (independently from their distribution), and they will be used to account for the number of ones. $P_{h}(A)$ is a distribution measure of $o$ ones over $v$ rows, whereas $P_{v}(A)$ is a distribution measure of $o$ ones over $b$ columns. Thus, for $A^{\prime} \in \mathcal{A}_{o}$ (the subset of all configurations with exactly $o$ ones), $P_{h}\left(A^{\prime}\right)=P_{h}^{\min }(o)$ if and only if the rows in $A^{\prime}$ are maximally uniform, and $P_{v}\left(A^{\prime}\right)=P_{v}^{\min }(o)$ if and only if the columns in $A^{\prime}$ are maximally uniform. For $o=u$, since admissibility conditions (5)-(6) enforce multiplicities $(v \mid o$ and $b \mid o$ ), these distributions are strictly uniform.

Likewise, let $p_{v}=P_{v}(A)$ be the number of vertical pairs in a given configuration $A$. Then, $Q(A)$ can be interpreted as a distribution measure of $p_{v}$ vertical pairs ("balls") over $f=\left(\begin{array}{l}v \\ 2\end{array}\right)$ pairs of rows ("bins"). In this sense, a maximally distributed configuration is called maximally balanced, with lower bound $Q^{\min }\left(p_{v}\right)$. For $\left(p_{v}, f\right)$ such that $f \mid p_{v}$ (multiplicity condition), a maximally balanced configuration is said to be strictly balanced. Yet, as long as $p_{v}$ is the same, balance may equally be reached by a configuration with fewer ones unevenly distributed over columns, or by a configuration with more ones which are better distributed. Given a number $o$ of ones, since $Q^{\min }\left(p_{v}\right)$ is non-decreasing, the lower bound on $Q(A)$ over $\mathcal{A}_{o}$, denoted $Q^{\min }(o)$, is

$$
Q^{\min }(o)=Q^{\min }\left(P_{v}^{\min }(o)\right) .
$$

Since the complementary configuration of a $(v, b, u)$-BIBD is a $(v, b, v b-u)$ BIBD, the number $\bar{Q}(A)$ of quadruples of zeros can also be used as a measure of balance, with $\bar{o}=v b-o, \bar{Q}(A)=Q(\bar{A})$ and $\bar{A} \equiv\left[\bar{x}_{i j}\right]^{2}$.

\subsection{Characterizing BIBDs in terms of the distribution measures: Combina- torially optimal values}

The following theorem is a necessary and sufficient condition for the existence of a BIBD:

Theorem 4 Given a set $(v, b, u)$ of admissible parameters, a configuration $A^{*} \in \mathcal{A}-v \times b$ is the incidence matrix of $a(v, b, u)-B I B D$ if and only if

\footnotetext{
${ }^{2}$ For $P_{t}, P_{h}$ and $P_{v}$, minimization in terms of ones is equivalent to minimization in terms of zeros, thus the corresponding complementary measures are not necessary. This is not so for measure $Q$, as discussed in Section 3.1.
} 


$$
\begin{aligned}
& U\left(A^{*}\right)=u \quad \text { and } \\
& Q\left(A^{*}\right)=Q^{\min }(u) .
\end{aligned}
$$

Proof. Let $A_{1}$ be a configuration corresponding to a BIBD. Then, by definition, $A_{1}$ must have the right number of ones, must have strictly uniform columns and must be strictly balanced. The first fact simply states that $U\left(A_{1}\right)=u$. From this and the second fact it follows that the number of vertical pairs is given by $P_{v}\left(A_{1}\right)=P_{v}^{\min }(u)$. The third fact, balance, enforces maximal distribution of the available pairs $Q\left(A_{1}\right)=Q^{\min }\left(P_{v}\left(A_{1}\right)\right)=Q^{\min }\left(P_{v}^{\min }(u)\right)$ which, by equation (17), leads to (19).

On the other hand, let $A_{1}$ be a configuration satisfying (18) and (19). The first equation states that $A_{1}$ has the right number of ones and, since $Q\left(A_{1}\right)$ reaches its lower bound for $u$ ones, it follows that $A_{1}$ is maximally balanced. The number of vertical pairs involved must satisfy $P_{v}\left(A_{1}\right) \geq P_{v}^{\min }(u)$ but, by equation (17), only the equality is possible, otherwise $Q\left(A_{1}\right)$ would be higher than expressed in $(19)^{3}$. Therefore, $A_{1}$ has maximally uniform columns. Since the parameter set is admissible, maximalities are satisfied in the strict sense; and, from strict balance, strictly uniform columns and the admissibility conditions it follows that rows are strictly uniform as well, thus completing the definition of BIBD.

A configuration $A^{*}$ satisfying conditions (18) and (19) is said to be a combinatorially optimal configuration.

Expanded according to (12) and (16), Theorem 4 constitutes the shortest definition of a BIBD in terms of the variables $x_{i j}$.

In the following, the assumption of optimality denotes the assumption that conditions 1-i to 1-iv in Definition 1 hold, even if no configuration exists that satisfies them, and the term optimal value refers to the value of an expression under such assumption (i.e., the value that the expression would take on a BIBD, if any existed).

For $M \in\left\{U, P_{t}, P_{v}, P_{h}, Q, \bar{Q}\right\}$, let $M^{\text {min }}(o)$ denote the lower bound of $M$ over $\mathcal{A}_{o}$, and let $\Delta M^{\mathrm{min}}(o)$ denote its increment, as defined in (11). Under the assumption of optimality, optimal values $M^{*}$ satisfy $M^{*}=\Delta M^{\min }(o)$, whereas optimal increments are defined as

$$
\Delta^{-} M^{*} \equiv \Delta M^{\min }(u-1)
$$

\footnotetext{
${ }^{3}$ This is only true for $\lambda>0$, which is in accordance with the definition of BIBD. Otherwise, the number of quadruples can remain zero even after adding vertical pairs $\left(Q^{\min }(u)=0, u=0, \ldots, f\right)$.
} 
Table 1

Given a set $(v, b, u)$ of admissible parameters, optimal values and optimal increments for $U, P_{t}, P_{h}, P_{v}, Q$ and $\bar{Q}$, expressed in terms of the descriptors of the design (with $\bar{k}=v-k$ and $\bar{\lambda}=b+\lambda-2 r)$.

\begin{tabular}{|c|c|c|c|}
\hline$M$ & $M^{*}$ & $\Delta^{-} M^{*}$ & $\Delta^{+} M^{*}$ \\
\hline \hline$U$ & $u$ & 1 & 1 \\
\hline$P_{t}$ & $\left(\begin{array}{l}u \\
2\end{array}\right)$ & $u-1$ & $u$ \\
\hline$P_{h}$ & $v\left(\begin{array}{l}r \\
2\end{array}\right)$ & $r-1$ & $r$ \\
\hline$P_{v}$ & $b\left(\begin{array}{l}k \\
2\end{array}\right)$ & $k-1$ & $k$ \\
\hline$Q$ & $f\left(\begin{array}{l}\lambda \\
2\end{array}\right)$ & $(k-1)(\lambda-1)$ & $k \lambda$ \\
\hline $\bar{Q}$ & $f\left(\begin{array}{l}\bar{\lambda} \\
2\end{array}\right)$ & $-\bar{k} \bar{\lambda}$ & $-(\bar{k}-1)(\bar{\lambda}-1)$ \\
\hline
\end{tabular}

$$
\Delta^{+} M^{*} \equiv \Delta M^{\min }(u) .
$$

Under the assumption of optimality, admissibility conditions hold, thus enforcing multiplicities. In that case, for each of the above measures, the expressions for the lower bounds (from 10) and increments (from 11) are simple, as shown in Table 1.

\subsection{The parametric cost function $F$}

We are now in condition to define a generic combinatorial cost function for the generation of BIBDs. Let

$$
F=\rho_{u} U+\rho_{t} P_{t}+\rho_{h} P_{h}+\rho_{v} P_{v}+\rho_{q} Q+\rho_{\bar{q}} \bar{Q},
$$

be a linear combination of the measures defined in Section 2.1 (the dependence on $A$ is omitted hereafter for the sake of clarity). Then, a particular function is univocally specified by the set of its composition coefficients

$$
F\left(\rho_{u}, \rho_{t}, \rho_{h}, \rho_{v}, \rho_{q}, \rho_{\bar{q}}\right) .
$$

Theorem 5 Given a set $(v, b, u)$ of admissible parameters, the measure $F$ in equation (22) is a combinatorial cost function for the generation of BIBDs if

$$
\begin{array}{r}
\rho_{q}>0 \\
\rho_{t}, \rho_{h}, \rho_{v}, \rho_{\bar{q}} \geq 0
\end{array}
$$

and $\rho_{u}$ is such that 


$$
\begin{aligned}
& \Delta^{-} F^{*}<0 \\
& \Delta^{+} F^{*}>0 .
\end{aligned}
$$

Its global minimum is

$$
\begin{aligned}
F^{*} & =F^{\min }(u)= \\
& =\rho_{u} U^{*}+\rho_{t} P_{t}^{*}+\rho_{h} P_{h}^{*}+\rho_{v} P_{v}^{*}+\rho_{q} Q^{*}+\rho_{\bar{q}} \bar{Q}^{*},
\end{aligned}
$$

with the optimal values given in Table 1.

Proof. Since all lower bounds are non-concave, the drawing of $F^{\min }(o)$ must have a single valley, and multiplicity conditions (derived from parameter admissibility) guarantee that the bottom of this valley is not flat. We can then apply (26)-(27) and use $\rho_{u}$ to set its global minimum at the desired point $(o=u)$. Condition $(24)$ is necessary to guarantee that Theorem 4 can be applied.

The family of cost functions defined by the above theorem has been experimentally validated on the set of all BIBDs of size $v b \leq 1000$. This was a by-product of a study where the generation of BIBDs was used as a benchmark for comparing the performance of several optimization strategies based on neural networks [2]. We omit the details here, since the main objective of this work is the extension of these cost functions to the case of non-admissible parameters, which is undertaken in the next section.

\section{A combinatorial extension of BIBDs: MBMUDs}

The general criterion of maximally uniform distribution (Definition 3) is used here as a generalization of the strictly uniform criterion applied in the definition of BIBDs (Definition 1). In other words, the admissibility constraints (5-7) on the design parameters are relaxed, and a super-class including BIBDs is defined for arbitrary $(v, b, u)$. In terms of combinatorial balance, Maximally Balanced Maximally Uniform Designs $(v, b, u)$-MBMUDs are the closest possible approximation to BIBDs in cases where the parameter set is not admissible.

Definition 6 (MBMUD) We say that $A \in \mathcal{A}-v \times b$ is the incidence matrix of a Maximally Balanced Maximally Uniform Design (MBMUD) with parameters $(v, b, u)$ and descriptors $\left[(r, k, \lambda),\left(v_{0}, b_{0}, f_{0}\right)\right]$, with $0 \leq u \leq v b$, if and only if the following properties are fulfilled:

i) Right number of ones: $o=u$. 
A1, a $(6,10,27)$-MBMUD

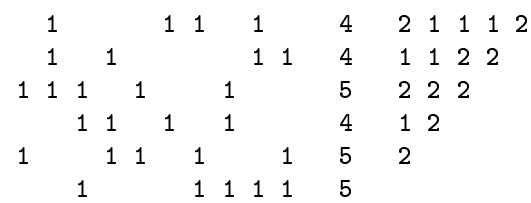

$\begin{array}{llllllllllll}2 & 3 & 3 & 3 & 2 & 2 & 3 & 3 & 3 & 3 & 27\end{array}$
A2, non uniform rows

\begin{tabular}{|c|c|c|c|c|c|c|c|c|c|c|}
\hline & & 1 & 1 & 11 & 1 & & 1 & 5 & 2 & \\
\hline 1 & 1 & 1 & & 1 & & & & 4 & 2 & \\
\hline & 1 & & 11 & 11 & & & & 4 & 1 & \\
\hline 1 & & 1 & 1 & & & & 1 & 4 & 2 & \\
\hline 1 & 1 & & & & & 1 & 1 & 4 & 2 & \\
\hline 11 & & & 11 & 1 & 1 & 1 & & 6 & & \\
\hline
\end{tabular}

Fig. 1. Examples of $(6,10,27)$-configurations (ones of the incidence matrix, row and column sizes, number of ones and triangular matrix of row concurrences). A1 is a $(6,10,27)$-MBMUD with descriptors $[(4,2,1)(3,7,9)]$. In A2, although columns and concurrences are maximally uniform, the number of ones per row takes three different values.

ii) Maximally uniform rows: There are $v_{0}$ rows with $r+1$ ones and the remaining rows have $r$ ones.

iii) Maximally uniform columns: There are $b_{0}$ columns with $k+1$ ones and the remaining columns have $k$ ones.

iv) Maximum balance: There are $f_{0}$ pairs of rows with concurrence $\lambda+1$ and the remaining pairs have concurrence $\lambda_{i k}=\lambda$.

This time, property 6-ii can no longer be derived from 6-iii and 6-iv, as was the case for BIBDs. This fact is shown in Figure 1. According to the criterion of maximal distribution, parameters and descriptors are now related by

$$
\begin{array}{cl}
r=\lfloor u / v\rfloor & v_{0}=u \bmod v \\
k=\lfloor u / b\rfloor & b_{0}=u \bmod b \\
\lambda=\left\lfloor p_{v}^{*} / f\right\rfloor & f_{0}=p_{v}^{*} \bmod f
\end{array}
$$

with $f=\left(\begin{array}{l}v \\ 2\end{array}\right)$ the number of pairs of rows, and $p_{v}^{*}=b\left(\begin{array}{l}k \\ 2\end{array}\right)+b_{0} k$ the optimal number of vertical pairs. Notice that maximum balance can be reached by a configuration with non-uniform columns, as long as it has the optimal value of vertical pairs (Section 3.1).

For $(v, b, u)$ such that all multiplicity conditions hold, all maximal distributions are achieved in the strict sense, the second set of descriptors $\left(v_{0}, b_{0}, f_{0}\right)$ is $(0,0,0)$, and the corresponding configuration is a BIBD. For $(v, b, u)$ such that $v \mid u$ and $b \mid u$, the corresponding configuration is a RGD $[6,17]$. When only $b \mid u$, the corresponding configuration is a SRDG [5], which coincides with a NBIBD of Type I [3].

The above definition is the natural consequence of applying cost function $F(22)$ to a set of non-admissible parameters. In fact, if the coefficients of $F$ satisfy some additional constraints (Section 3.2) then optima under $F$ correspond to MBMUDs. In the next sections we use the generalized criterion of maximally uniform distribution to derive optimal values for the distribution 
Table 2

Optimal values and increments for the measures $U, P_{t}, P_{h}, P_{v}$ and $Q$, in terms of the descriptors $\left[(r, k, \lambda),\left(v_{0}, b_{0}, f_{0}\right)\right]$ of a MBMUD with parameters $(v, b, u)$. Measure $\bar{Q}$ has been omitted since it can no longer be used for MBMUD generation in the general case (see text).

\begin{tabular}{|c|c|c|c|}
\hline$M$ & $M^{*}$ & $\Delta^{-} M^{*}$ & $\Delta^{+} M^{*}$ \\
\hline \hline$U$ & $u$ & 1 & 1 \\
\hline$P_{t}$ & $\left(\begin{array}{l}u \\
2\end{array}\right)$ & $u-1$ & $u$ \\
\hline$P_{h}$ & $v\left(\begin{array}{l}r \\
2\end{array}\right)+v_{0} r$ & $\left\{\begin{array}{l}r-1 \text { if } v_{0}=0 \\
r \text { if } v_{0} \neq 0\end{array}\right.$ & $r$ \\
\hline$P_{v}$ & $b\left(\begin{array}{l}k \\
2\end{array}\right)+b_{0} k$ & $\left\{\begin{array}{l}k-1 \text { if } b_{0}=0 \\
k \text { if } b_{0} \neq 0\end{array}\right.$ & $k$ \\
\hline$Q$ & $f\left(\begin{array}{l}\lambda \\
2\end{array}\right)+f_{0} \lambda$ & $\left\{\begin{array}{c}(k-1)(\lambda-1)+f_{0} \\
\text { if } b_{0}=0, f_{0}<k-1 \\
k-1) \lambda \text { if } b_{0}=0, f_{0} \geq k-1 \\
k(\lambda-1)+f_{0} \text { if } b_{0} \neq 0, f_{0}<k \\
k \lambda \text { if } b_{0} \neq 0, f_{0} \geq k\end{array}\right.$ & $\left\{\begin{array}{c}k \lambda \text { if } f_{0} \leq f-k \\
k(\lambda+1)-\left(f-f_{0}\right) \\
\text { if } f_{0}>f-k\end{array}\right.$ \\
\hline
\end{tabular}

measures of Section 2.1, and MBMUDs are characterized in terms of them.

\subsection{The distribution measures applied to MBMUDs: Optimal values}

In the case of MBMUDs, the assumption of optimality refers to Definition 6 . Under such assumption, optimal values and optimal increments are listed in Table 2 .

Theorem 7 Given a set $(v, b, u)$ of parameters, with $0 \leq u \leq v b$, a configuration $A^{*} \in \mathcal{A}-v \times b$ is the incidence matrix of a $(v, b, u)-M B M U D$ if and only if

$$
\begin{aligned}
U(A) & =u, \\
P_{h}(A) & =P_{h}^{*}, \\
P_{v}(A) & =P_{v}^{*} \quad \text { and } \\
Q(A) & =Q^{*} .
\end{aligned}
$$

Proof. If $A^{*}$ is a MBMUD, the above conditions follow. In the other sense of the implication, conditions (32) and (35) together enforce balance, but since $\lambda$ is now unrestricted, the condition on $P_{v}$ must also be fulfiled to enforce column uniformity (when $Q^{*}=0$ ). Besides, since column-uniformity and balance no longer guarantee row-uniformity (Figure 1), the condition on $P_{h}$ must also be 
imposed in the general case.

For some parameter sets it is easy to show that no MBMUD can exist. One such situation is the case when

$$
b_{0}=0, f_{0}=0, \text { and } v_{0} \neq 0 .
$$

If there exists a configuration satisfying the first two conditions then it must have strictly uniform rows, in contradiction with the fact that $v_{0} \neq 0$ (multiplicity for rows is not satisfied). An example of this situation is the set $(6,10,40)$ with descriptors $[(6,4,4),(4,0,0)]$, which is particularly interesting since, as reported in Section 3.3, there do exist MBMUDs with the complementary parameters $(6,10,20)$. Thus, given two sets of complementary parameters $(v, b, u)$ and $(v, b, v b-u)$, the existence of a $(v, b, u)$-MBMUD does not imply that its complementary configuration is a $(v, b, v b-u)$-MBMUD. In fact, it does not even imply that there exists any $(v, b, v b-u)$-MBMUD.

Proposition 8 Given a set $(v, b, u)$ of parameters, if

$$
v_{0}=0 \text { or }\left(f_{0}=0 \text { and }\left(v_{0}=1 \text { or } v_{0}=v-1\right)\right) \text {, }
$$

then, if there exists a $(v, b, u)-M B M U D$, its complementary configuration is also a MBMUD with parameters $(v, b, v b-u)$.

Proof. If condition (37) is satisfied, the casuistry for

$$
\bar{\lambda}_{i k}=b+\lambda_{i k}-r_{i}-r_{k}
$$

yields at most two different values.

Of course, admissible parameters for BIBDs satisfy (37).

For measures $U, P_{t}, P_{h}$ and $P_{v}$ it is easy to verify that optimality in a given configuration $A$ implies optimality in its complementary configuration $\bar{A}$. Thus, from Theorem 7 and the above examples it follows that optimality on $Q$ does not imply optimality on $\bar{Q}$ (i.e., $\left.Q(A)=Q^{*} \nRightarrow Q(\bar{A})=\bar{Q}^{*}\right)$.

The next result states a relationship with the dual configuration space $\mathcal{A}^{\prime}-b \times v$. The optimal lower bound on $Q$ was derived by assuming column uniformity and balance, that is, by taking $Q^{*}=Q^{\min }(u)=Q^{\min }\left(P_{v}^{\min }(u)\right)$. Let us call this bound the balanced lower bound on $Q$ for balanced, column-uniform 
configurations with $u$ ones, and let us denote it by $Q_{b}$. Then (Table 2),

$$
Q_{b}=f\left(\begin{array}{l}
\lambda \\
2
\end{array}\right)+f_{0} \lambda
$$

Yet, using a dual point of view, the number of quadruples in a configuration $A$ can also be interpreted as a distribution measure of the horizontal pairs ("balls") in $A$ over pairs of columns ("bins"). From this point of view, let $\mu_{j l}$ be the concurrence between columns $j$ and $l$. Then, for any configuration $A$,

$$
Q(A)=\sum_{j} \sum_{l \neq j}\left(\begin{array}{c}
\mu_{j l} \\
2
\end{array}\right)
$$

We say that a configuration is maximally linked when concurrences between pairs of columns are maximally uniform. As usual, with $p_{h}$ the number of horizontal pairs and $g=\left(\begin{array}{l}b \\ 2\end{array}\right)$ the number of pairs of columns, we say that the configuration is strictly linked when the multiplicity $g \mid p_{h}$ holds. Then, proceeding as before, the linked lower bound on $Q$ for linked, row-uniform configurations with $u$ ones is

$$
Q_{l}=g\left(\begin{array}{l}
\mu \\
2
\end{array}\right)+g_{0} \mu
$$

with $\mu=\left\lfloor p_{h}^{*} / g\right\rfloor, g_{0}=p_{h}^{*} \bmod g$, and $p_{h}^{*}=v\left(\begin{array}{l}r \\ 2\end{array}\right)+v_{0} r$ the optimal number of horizontal pairs.

Theorem 9 A necessary condition for the existence of a $(v, b, u)-M B M U D$ is that $Q_{b}$ (39) and $Q_{l}$ (41) satisfy

$$
Q_{b} \geq Q_{l}
$$

Proof. Since both $Q_{b}$ and $Q_{l}$ are lower bounds on $Q$, both underlying assumptions (i.e., maximum balance and maximum linkage) can only be fulfilled simultaneously when $Q_{b}=Q_{l}$. Then, since MBMUDs are defined in terms of balance, (42) must hold.

For instance, designs with parameters $(6,10,40),(6,10,50)$ and $(6,10,51)$ do not exist (the first was already mentioned as an example of (36)). 
The following statements are derived from the preceding theorem:

- The dual of a maximally balanced design is a maximally linked configuration.

- If there exists a MBMUD with $Q_{b}>Q_{l}$, its dual is not a MBMUD.

- If there exists a $(v, b, u)$-MBMUD with $Q_{b}=Q_{l}$, then it is a maximally linked MBMUD, and its dual is a $(b, v, u)$-MBMUD.

And it also holds that

- If a MBMUD is square $(v=b)$, then $Q_{b}=Q_{l}$. But $Q_{b}=Q_{l}$ does not imply $v=b$.

As an existence condition, Theorem 9 is ineffective when $v=b$, it is hardly restrictive when $v<b$, but it is strongly restrictive for $v>b$. Fisher's condition, thus, seems to show up in a sort of relaxed way. For $b_{0}=0$ MBMUDs revert to type I NBIBDs. The reader is referred to [3] and [13] for further existence results.

\subsection{Cost function F for MBMUDs}

The constraints on the coefficients of the cost function $F$ when applied to MBMUDs depend on the descriptors $\left(v_{0}, b_{0}, f_{0}\right)$. In order to get a valid cost function for any MBMUD, we now consider the worst-case.

Theorem 10 Given a set $(v, b, u)$ of parameters, with $0 \leq u \leq v b$, the measure $F\left(\rho_{u}, \rho_{t}, \rho_{h}, \rho_{v}, \rho_{q}, \rho_{\bar{q}}\right)$ is a combinatorial cost function for the generation of $M B M U D s$ if

$$
\begin{array}{r}
\rho_{t}, \rho_{h}, \rho_{v}, \rho_{q}>0 \\
\rho_{\bar{q}}=0,
\end{array}
$$

and $\rho_{u}$ is such that

$$
\begin{aligned}
& \Delta^{-} F^{*}<0 \\
& \Delta^{+} F^{*}>0 .
\end{aligned}
$$

Its global minimum is

$$
\begin{aligned}
F^{*} & =F^{\min }(u)= \\
& =\rho_{u} U^{*}+\rho_{t} P_{t}^{*}+\rho_{h} P_{h}^{*}+\rho_{v} P_{v}^{*}+\rho_{q} Q^{*},
\end{aligned}
$$

with the optimal values given in Table 2. 
A3, unbalanced

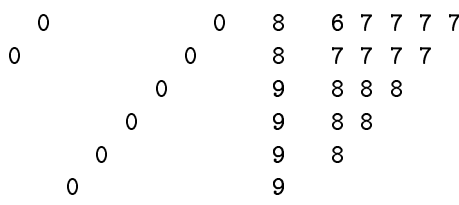

$6 \begin{array}{llllllllll}6 & 5 & 5 & 5 & 5 & 5 & 5 & 5 & 5 & 52\end{array}$

Fig. 2. Optimal distribution of zeros for a configuration with parameters $(6,10,52)$. All other alternatives are isomorphic. No other distribution preserves the uniformity of rows and columns, but concurrences are not balanced. Therefore, no $(6,10,52)$-MBMUD can exist.

Proof. From Theorem 7, the terms on $Q, P_{v}$ and $P_{h}$ must be present in a general-case cost function for MBMUDs, and the term on $\bar{Q}$ must be discarded, as discussed in relation to the complementary configuration. The worst case occurs for $(v, b, u)$ such that none of the multiplicity conditions holds. In this case, in order to avoid that $\Delta F^{\text {min }}$ be flat in the desired region, $P_{t}$ must be explicitly included, since it has a monotonically increasing increment. Then, since the resulting lower bound is convex, the minimum is set by adjusting $\rho_{u}$ so that (45)-(46) are satisfied.

\subsection{Experimental validation}

All MBMUDs in this section were generated using $F\left(\alpha_{u}, 1,9,3,7,0\right)$ and Simulated Annealing [8] as optimization technique. The dual search spaces $\mathcal{A}-6 \times 10$ and $\mathcal{A}^{\prime}-10 \times 6$ were thoroughly analysed in order to check the properties discussed, and results are summarized in Table 3.

A "pathological" situation was detected when the desired number of ones $u$ is close to the size $v b$ of the incidence matrix. If both row and column uniformities are to be preserved, the $v b-u$ zeros of the design should all be in different rows and columns, but then concurrences between rows would take three different values. This happens when

$$
\bar{u} \leq \max (v, b) \text { and } 1<v_{0}<v-1 \text {. }
$$

As an example, the case $(6,10,52)$ is shown in Figure 2. Besides these, and the non-existing cases derived from Theorem 9 and from equation (36), the only unsolved case was $(6,10,41)$. Although the size $v b$ was the same for them all, notice the differences in cost to the first solution obtained from problem to problem.

Finally, 30 other problems were tried with sizes similar to those of BIBDs $(v b<300)$. From 23 cases with $v \leq b, 19$ problems were solved, whereas from 
Table 3

Exploration of the search space $\mathcal{A}-6 \times 10$ (left), and its dual $\mathcal{A}^{\prime}-10 \times 6$ (right). With $u$ and $\bar{u}$ all possible number of ones are covered. In each case, $\hat{E}(\mathbf{z})$ is the estimated cost to the first solution (in iterations), and "Exists?" describes existence results. Non-existence is signaled with $\star$ for Theorem 9, $\circ$ for equation (36) and $\diamond$ for equation (48) (see text).

\begin{tabular}{|c|c|c|c|c|c|c|c|c|c|}
\hline & & \multicolumn{4}{|c|}{$\mathcal{A}-6 \times 10$} & \multicolumn{4}{|c|}{$\mathcal{A}^{\prime}-10 \times 6$} \\
\hline & & \multicolumn{2}{|c|}{$\operatorname{DME}(v, b, u)$} & \multicolumn{2}{|c|}{$\operatorname{DME}(v, b, \bar{u})$} & \multicolumn{2}{|c|}{$\operatorname{DME}(v, b, u)$} & \multicolumn{2}{|c|}{$\operatorname{DME}(v, b, \bar{u})$} \\
\hline$u$ & $\bar{u}$ & $\hat{E}(\mathbf{z})$ & Exists? & $\hat{E}(\mathbf{z})$ & Exists? & $\hat{E}(\mathbf{z})$ & Exists? & $\hat{E}(\mathbf{z})$ & Exists? \\
\hline 0 & 60 & 1.0 & & 1.0 & & 1.0 & & 1.0 & \\
\hline 1 & 59 & 1.0 & & 1.0 & & 1.0 & & 1.0 & \\
\hline 2 & 58 & 2.0 & & - & no $[\diamond]$ & 1.9 & & - & no $[\diamond]$ \\
\hline 3 & 57 & 2.0 & & - & no $[\diamond]$ & 2.0 & & - & no $[\diamond]$ \\
\hline 4 & 56 & 2.0 & & - & no $[\diamond]$ & 2.0 & & - & no $[\diamond, \star]$ \\
\hline 5 & 55 & 2.0 & & 2.0 & & 2.0 & & - & no $[\diamond, \star]$ \\
\hline 6 & 54 & 24.6 & & 2.8 & & 2.7 & & - & no $[\diamond, \star]$ \\
\hline 7 & 53 & 291.5 & & 2.6 & & 2.7 & & - & no $[\diamond, \star]$ \\
\hline 8 & 52 & 93.3 & & - & no $[\diamond]$ & 2.2 & & - & no $[\diamond]$ \\
\hline 9 & 51 & 39.1 & & - & no $[\star]$ & 2.6 & & 2.4 & \\
\hline 10 & 50 & 3.0 & & - & no $[\star]$ & 8.3 & & 4.1 & \\
\hline 11 & 49 & 4.4 & & 3.2 & & 133.3 & & - & no $[\star]$ \\
\hline 12 & 48 & 9.5 & & 7.4 & & 15.6 & & - & no $[\star]$ \\
\hline 13 & 47 & 13.9 & & 4.0 & & 3.1 & & - & no $[\star]$ \\
\hline 14 & 46 & 6.5 & & 75.5 & & 2.7 & & - & no $[\star]$ \\
\hline 15 & 45 & 3.7 & & 113.9 & & 2.7 & & - & no $[\star]$ \\
\hline 16 & 44 & 2.6 & & 123.6 & & 2.6 & & - & no $[\star]$ \\
\hline 17 & 43 & 2.7 & & 2520.2 & & 2.4 & & - & no $[\star]$ \\
\hline 18 & 42 & 16.7 & & 154.2 & & 2.5 & & - & no $[\star]$ \\
\hline 19 & 41 & 171.5 & & - & & 30.0 & & - & no $[\star]$ \\
\hline 20 & 40 & 6.3 & & - & no $[\circ, \star]$ & 8.6 & & 246.8 & \\
\hline 21 & 39 & 66.2 & & 1556.9 & & 83.4 & & 2487.5 & \\
\hline 22 & 38 & 27.1 & & 80.6 & & 133.8 & & - & no $[\star]$ \\
\hline 23 & 37 & 38.7 & & 180.0 & & - & no $[\star]$ & - & no $[\star]$ \\
\hline 24 & 36 & 10.8 & & 18.6 & & - & no $[\star]$ & - & no $[\circ, \star]$ \\
\hline 25 & 35 & 22.3 & & 67.3 & & - & no $[\star]$ & - & no $[\star]$ \\
\hline 26 & 34 & 21.9 & & 46.2 & & - & no $[\star]$ & - & no $[\star]$ \\
\hline 27 & 33 & 18.3 & & 86.6 & & - & no $[\star]$ & - & no $[\star]$ \\
\hline 28 & 32 & 23.5 & & 91.1 & & - & no $[\star]$ & - & no $[\star]$ \\
\hline 29 & 31 & 595.6 & & 274.6 & & - & no $[\star]$ & - & no $[\star]$ \\
\hline 30 & & 24.7 & & & & 4060.6 & & & \\
\hline
\end{tabular}


7 cases with $v>b$ only 3 problems were solved. Results are detailed in [1]. In all, MBMUD problems proved to be about as difficult as BIBDs.

\section{MBMUDs and statistical optimality: some experimental results}

\subsection{Optimality measures, optimization procedure and experimental set}

The statistical optimality criteria for block designs are usually defined in terms of the information matrix (or $C$-matrix). The generalized inverse of this matrix is proportional to the variance-covariance matrix for estimating the treatment effects.

For an arbitrary design with incidence matrix $A$, the $C$-matrix is given by [16]:

$$
C=R-A K^{-1} A^{T},
$$

where $R$ and $K$ are diagonal matrices with the number of ones per row and column, respectively.

Let $\mu_{i}$ be the non-zero eigenvalues of $C$, then the A- and D-optimality measures $\Phi_{A}$ and $\Phi_{D}$ are derived as follows:

$$
\begin{aligned}
& \Phi_{A}(A)=\sum 1 / \mu_{i}, \\
& \Phi_{D}(A)=1 / \prod \mu_{i} .
\end{aligned}
$$

In the experiments, we will be using these two statistically optimality measures to assess how informative is the proposed combinatorial cost function.

It is also important to define precisely the domain in which the optimization will be carried out. Let $\mathcal{A}(v, b, u)$ be the set of $v \times b$ configurations with exactly $u$ ones, uniform rows and uniform columns. The search strategy of our optimization algorithm explores only configurations in $\mathcal{A}(v, b, u)$, by updating at each step square-wise arrangements of units from $(10,01)$ to $(01,10)[10]$. In this way, the first subgoals of our cost function (number of ones, ones per row and ones per column) are guaranteed by construction, and the only term that needs to be minimized is the number of quadruples. Combinatorially optimal designs over $\mathcal{A}(v, b, u)$ according to this criterion will be hereafter called Qoptimal designs.

For the interested reader, the source code and executable version of our algorithm are available at [4]. 
The set of designs selected to perform our experiments is based on the set explored in [3], extended with several non-NBIBD MBMUDs. The list of designs considered appears in Table 4, classified as BIBDs, RGDs, type-I NBIBDs (or SRGDs), type-II NBIBDs, non-existing NBIBDs, and non-NBIBD MBMUDs.

\subsection{Optimality behaviour of the proposed combinatorial cost function}

The first set of experiments was aimed at determining the relation of the proposed combinatorial cost function with the $\Phi_{A^{-}}$and $\Phi_{D^{-}}$statistical optimality measures. For all designs, the space $\mathcal{A}(v, b, u)$ was sampled with a uniform distribution and the values of the three measures were recorded for each configuration. As an example of the results, Figure 3 plots the $\Phi_{A}(A)$ values as a function of the $Q(A)$ values obtained for a particular design. An almost identical plot was obtained for $\Phi_{D}(A)$ versus $Q(A)$, and similar grafs came out for the other designs as well. The discrete nature of the $Q$ function versus the continuous nature of the statistical optimality measures is apparent. For every value of $Q$ there are several values of $\Phi_{A}(A)$ (i.e., there exist several non-isomorphic configurations with the same $Q$ ), but the overall tendency of the plot is clearly monotonic. Some of the strides overlap, meaning that it is sometimes possible to minimize $Q$ while actually increasing $\Phi_{A}$, but only for $Q$ values larger than $Q^{*}$. In fact, for all the designs tested (including the non-

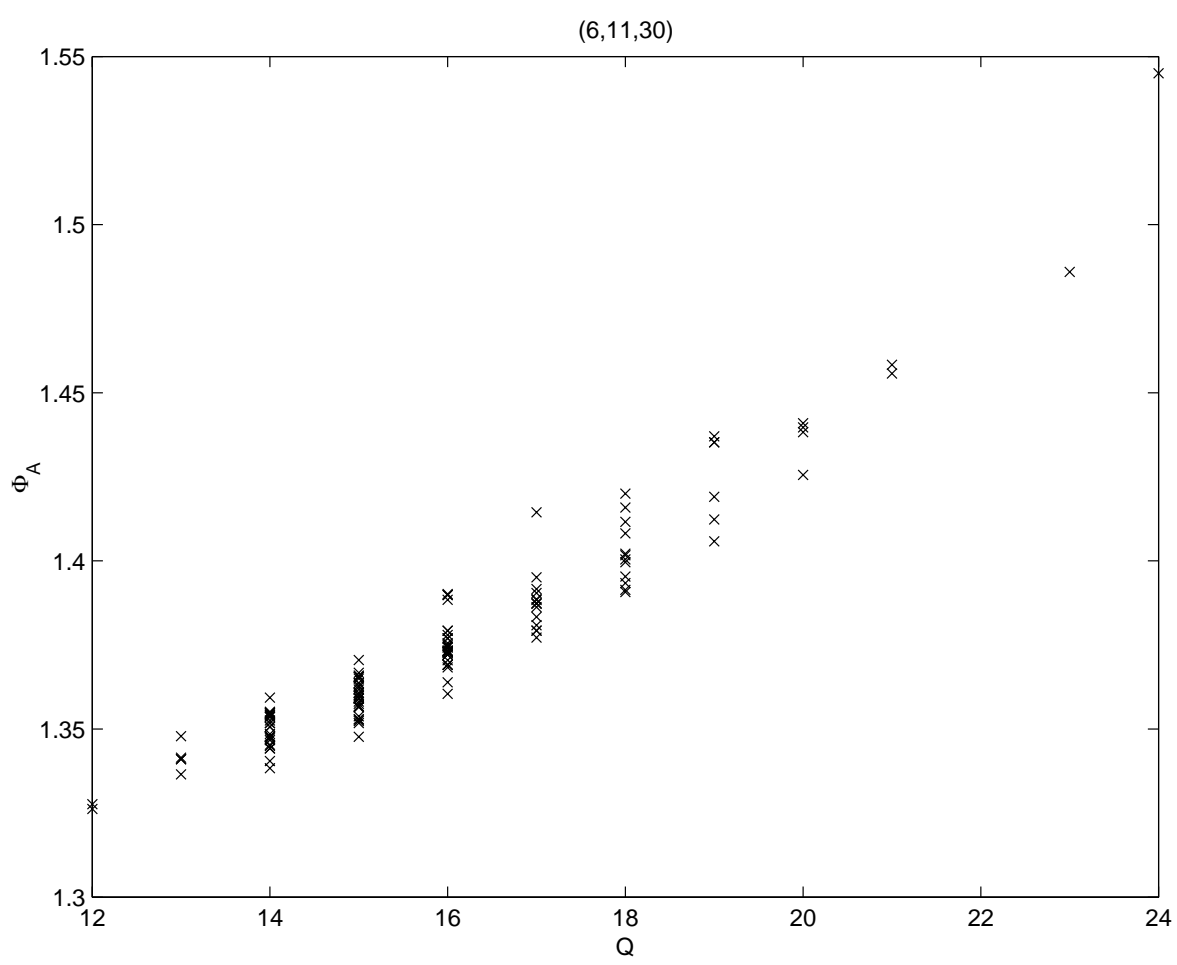

Fig. 3. Plot of $\Phi_{A}(A)$ vs $Q(A)$ for uniform configurations with parameters $(6,11,30)$. 
existing ones), the leftmost strides didn't overlap, and had the lowest values. In the case of BIBDs, since they are Q-,A- and D-optimal, the leftmost stride reduces to a single point.

In general terms, thus, combinatorial optimality was found to be a necessary but not sufficient condition for statistical optimality. For small $u$ this leads to complete enumeration of competing concurrence patterns to determine statistical optimality as in [12], while for larger $u$ it tells us that statistical optimality is generally much harder to establish than combinatorial optimality.

The next set of experiments consisted of performing 30 descents of the optimization procedure on each of the three landscapes, i.e., using measures $Q(A)$, $\Phi_{A}(A)$ and $\Phi_{D}(A)$, respectively. The average values of $\Phi_{A}$ attained in the descents on $Q(A)$ and $\Phi_{A}(A)$ are recorded in the second and third columns of Table 4 , while the average values of $\Phi_{D}$ attained in the descents on $Q(A)$ and $\Phi_{D}(A)$ are recorded in the fifth and sixth columns of the same table.

At first glance one sees that the values of $\Phi_{A}$ obtained by descending on the $Q$ and $\Phi_{A}$-landscapes are very similar. And the same observation holds for the $\Phi_{D}$ values. Hence, the proposed cost function has minima close to those of the usual A- and D- statistical optimality measures. More precisely, we observe that there is a perfect match in the cases of BIBDs and type-II NBIBDs, and in several of the RGDs and type-I NBIBDs as well. As should be expected, for designs coincident with those listed in Table 1 of [3], the values of $\Phi_{A}$ and $\Phi_{D}$ are concordant with the efficiency bounds recorded there.

Some other observations are worth mentioning. The number of Q-optimal designs obtained was high for the three landscapes (around $98 \%$ of the descents). Type-II NBIBD settings were of course not Q-optimal, but in this case our procedure actually converged to Type-II NBIBD. On the contrary, non-existing NBIBDs converged to configurations with 3 different concurrence values for the same row. Yet, best $Q$ s are only 2 or 3 steps away from $Q^{*}$.

$Q(A)$ showed a consistently much lower computational cost (with savings ranging from $50 \%$ to $90 \%$ ) for two reasons. On the one hand, the computational complexity of each function evaluation is much lower, as compared with the statistical optimality measures, which require a singular value decomposition per evaluation. In fact, $Q(A)$ is computed incrementally by considering the joint effect of a $(10,01) \rightarrow(01,10)$ transformation (the gradient has an explicit closed form, which permits avoiding to really "evaluate" the function each time). On the other hand, and as an experimental observation, the number of iterations per descent was almost always smaller in the case of $Q(A)$, while reaching minima that were similar in quality.

Most A- and D-local minima were also Q-local minima but not the other way around. This fact suggested a third set of experiments. Local minima of 
Table 4

Average values of $\Phi_{A}$ (respectively, $\Phi_{D}$ ) attained in the following three situations: after 30 descents on the $\Phi_{A}$-landscape (resp. $\Phi_{D}$-landscape), after 30 descents on the $Q$-landscape, and by minimizing $\Phi_{A}\left(\operatorname{resp} . \Phi_{D}\right)$ after the descents on $Q$.

\begin{tabular}{|c|c|c|c|c|c|c|}
\hline \multirow[t]{2}{*}{$(v, b, u)$} & \multicolumn{3}{|c|}{$\Phi_{A}$ values } & \multicolumn{3}{|c|}{$10000 * \Phi_{D}$ values } \\
\hline & $\Phi_{A}$-landscape & $Q$-landscape & $Q$-initialization & $\Phi_{D}$-landscape & $Q$-landscape & $Q$-initialization \\
\hline \multicolumn{7}{|l|}{ BIBDs } \\
\hline$(6,10,30)$ & 1.2500 & 1.2500 & 1.2500 & 9.7656 & 9.7656 & 9.7656 \\
\hline$(6,15,60)$ & 0.5556 & 0.5556 & 0.5556 & 0.1694 & 0.1694 & 0.1694 \\
\hline \multicolumn{7}{|l|}{ RGDs } \\
\hline$(6,3,12)$ & 2.8333 & 2.8333 & 2.8333 & 555.5556 & 555.5556 & 555.5556 \\
\hline$(6,4,12)$ & 0.0030 & 0.0032 & 0.0030 & 1.0404 & 1.0547 & 1.0404 \\
\hline$(6,6,18)$ & 2.1250 & 2.1250 & 2.1250 & 131.5566 & 131.8359 & 131.5566 \\
\hline$(6,6,24)$ & 1.3987 & 1.3988 & 1.3987 & 16.8310 & 16.8327 & 16.8298 \\
\hline$(6,8,24)$ & 1.5871 & 1.5871 & 1.5871 & 30.9917 & 30.9917 & 30.9917 \\
\hline$(6,9,36)$ & 0.9286 & 0.9288 & 0.9286 & 2.1939 & 2.1947 & 2.1939 \\
\hline$(6,12,36)$ & 1.0490 & 1.0491 & 1.0490 & 3.9941 & 3.9945 & 3.9942 \\
\hline$(6,12,48)$ & 0.6952 & 0.6952 & 0.6952 & 0.5183 & 0.5183 & 0.5183 \\
\hline \multicolumn{7}{|c|}{ Type-I NBIBDs } \\
\hline$(6,3,9)$ & 0.0051 & 0.0054 & 0.0054 & 6.3333 & 7.5000 & 6.3333 \\
\hline$(6,7,21)$ & 1.8533 & 1.8556 & 1.8464 & 63.7996 & 63.9943 & 63.5616 \\
\hline$(6,9,27)$ & 1.4184 & 1.4178 & 1.4113 & 17.4072 & 17.3980 & 17.1213 \\
\hline$(6,11,33)$ & 1.1500 & 1.1500 & 1.1500 & 6.2500 & 6.2500 & 6.2500 \\
\hline$(6,14,56)$ & 0.5972 & 0.5972 & 0.5972 & 0.2411 & 0.2411 & 0.2411 \\
\hline$(6,16,64)$ & 0.5222 & 0.5222 & 0.5222 & 0.1235 & 0.1235 & 0.1235 \\
\hline \multicolumn{7}{|c|}{ Type-II NBIBDs } \\
\hline$(6,2,8)$ & 0.0052 & 0.0052 & 0.0052 & 6.6667 & 6.6667 & 6.6667 \\
\hline$(6,13,52)$ & 0.6438 & 0.6438 & 0.6438 & 0.3501 & 0.3501 & 0.3501 \\
\hline \multicolumn{7}{|c|}{$\begin{array}{c}\text { Non-existing } \\
\text { NBIBDs } \\
\end{array}$} \\
\hline$(6,4,16)$ & 2.2038 & 2.2139 & 2.2029 & 143.9496 & 144.9410 & 143.7103 \\
\hline$(6,5,15)$ & 2.7049 & 2.7272 & 2.7045 & 380.6391 & 385.6167 & 381.7127 \\
\hline$(6,5,20)$ & 1.7167 & 1.7228 & 1.7166 & 44.4444 & 44.7197 & 44.4387 \\
\hline$(6,7,28)$ & 1.2125 & 1.2131 & 1.2125 & 8.0058 & 8.0127 & 8.0053 \\
\hline$(6,8,32)$ & 1.0549 & 1.0554 & 1.0549 & 4.0537 & 4.0564 & 4.0539 \\
\hline$(6,10,40)$ & 0.8404 & 0.8406 & 0.8404 & 1.3135 & 1.3138 & 1.3135 \\
\hline$(6,11,44)$ & 0.7623 & 0.7625 & 0.7623 & 0.8112 & 0.8116 & 0.8113 \\
\hline \multicolumn{7}{|c|}{$\begin{array}{l}\text { Non-NBIBD } \\
\text { MBMUDs }\end{array}$} \\
\hline$(6,6,22)$ & 1.6031 & 1.6048 & 1.6031 & 31.7618 & 31.8115 & 31.7676 \\
\hline$(6,7,23)$ & 1.5928 & 1.5954 & 1.5924 & 31.2000 & 31.3085 & 31.2487 \\
\hline$(6,8,23)$ & 1.7116 & 1.7157 & 1.7088 & 43.9096 & 44.1675 & 43.7948 \\
\hline$(6,11,30)$ & 1.3197 & 1.3276 & 1.3179 & 12.7065 & 12.9025 & 12.6774 \\
\hline$(6,14,63)$ & 0.5115 & 0.5116 & 0.5115 & 0.1113 & 0.1114 & 0.1113 \\
\hline
\end{tabular}


descents with $Q$ were used as starting points for subsequent descents with $\Phi_{A}$ and $\Phi_{D}$. The results are recorded in the fourth and seventh columns of Table 4. Concerning the $\Phi_{A}$ values, in most cases the same result obtained with the direct minimization of $\Phi_{A}$ is reached, but note that in eight cases, this $Q$ initialization leads to a lower value and only in the case $(6,3,9)$ the resulting value is slightly higher. For the $\Phi_{D}$ values, the general trend is very similar, although the magnitude of the differences looks larger because the absolute values are larger. The success of the $Q$-initialization seems to suggest that the minima of $Q$ are located in areas containing "good" local minima of $\Phi_{A}$ and $\Phi_{D}$. By "good" we mean that they are lower than the average local minima in the entire $\Phi_{A^{-}}$and $\Phi_{D^{-}}$landscapes. This use of the $Q$ function as initialization deserves further consideration, since it might lead to better quality results at a reduced computational cost.

As a final comment let us mention that, besides the special behaviour of BIBDs, no other differences were observed between the different classes of designs. This somehow supports the validity of the MBMUD generalization.

\section{Conclusions}

This paper has discussed the generation of block designs as a combinatorial optimization problem, by defining a family of combinatorial cost functions that enforce the maximal distribution of ones in the incidence matrix of the design, for rows, columns and balance. Parameter admissibility on the independent $(v, b, u)$ parameters enforces single distinct values for the $[r, k, \lambda]$ design descriptors, leading to strictly uniform distributions, in all senses, i.e., leading to BIBDs.

The combinatorial optimization approach was then extended to arbitrary $(v, b, u)$, leading to the definition of Maximally Balanced Maximally Uniform Designs $(v, b, u)$-MBMUDs that, being maximally distributed, allow two distinct consecutive values for each of their descriptors $[r, r+1 ; k, k+1 ; \lambda, \lambda+1]$.

From a practical point of view, using $(v, b, u)$ as independent parameters makes sense since $v$ is the number of treatments under study, $b$ is the number of uniform blocks available, and $u$, the number of plots, is a measure of the resources that are necessary to perform the experiment (assume, for instance, a constant cost per trial). For arbitrary $(v, b, u)$, and in terms of combinatorial balance, MBMUDs are as similar as possible to BIBDs and, unlike pseudosolutions, they have a well-defined structure.

Through experimentation, we found that combinatorial optimality is apparently a necessary but not sufficient condition for statistical optimality. This 
is consistent with what has been known for binary designs in the restricted combinatorial class allowing just equisized blocks, as summarized in conjecture (3) of [15], page 60 . We also found that the proposed combinatorial cost function has a monotonic relation with A- and D- statistical optimality, while its computational cost is much lower. Thus, it can be used directly as an approximation of A- and D-efficiencies, or either serve to initialize any algorithm based on a statistical optimality criterion in order to speed up the minimization. Preliminary results suggest that, by using this initialization, local optimization procedures based on a statistical criterion may reach more efficient designs.

\section{Acknowledgements}

The authors would like to thank the editor and the anonymous referees for their thorough and accurate comments and sensible suggestions, that have greatly contributed to the current version of this paper.

\section{References}

[1] Bofill P., "Optimizing neural networks for the generation of block designs", PhD dissertation, Technical Report UPC-DAC-1997-76, November 1997.

[2] Bofill P. and Torras C., "Neural cost functions and search strategies for the generation of block designs: an experimental evaluation", Intl. Journal of Neural Systems, 11(2), 187-202, 2001.

[3] Cheng C.-S. and Wu C.-F., "Nearly balanced incomplete block designs", Biometrika, 68(2), 493-500, 1981.

[4] http://people.ac.upc.es/pau/mbmuds

[5] Jacroux M., "Some sufficient conditions for the type I optimality of block designs", Journal of Statistical Planning and Inference, 11, 385-398, 1985.

[6] John J.A and Mitchell T.J., "Optimal incomplete block designs", J. Roy. Statist. Soc. Ser. B, 39, 39-43, 1977.

[7] Kiefer J., "Construction and optimality of generalized youden designs", Srivastava J.N. (Ed.), A Survey of Statistical Design and Linear Models, NorthHolland, Amsterdam, 333-353, 1975.

[8] Kirkpatrik S., Gelart Jr. and Vecchi M.P., "Optimization by simulated annealing", Science, 220(671), 671-680, 1983.

[9] Kunert J., "Optimality of Block Designs with Variable Block Sizes and Random Block Effects", Metrika, 41, 71-81, 1994. 
[10] Mathon R., "Sa2des: A 2- $(\mathrm{v}, \mathrm{k}, \lambda)$ design generator, using simulated annealing search (Version 1.1d)", Toronto, 1989.

[11] Mathon R. and Rosa A., "2- $(v, k, \lambda)$ designs of small order", The CRC Handbook of Combinatorial Designs, CRC Press, 3-41, 1996.

[12] Mitchell T.J. and John J.A., "Optimal incomplete block designs", Oak Ridge Tech. report cited in [6], 1976.

[13] Morgan J.P. and Srivastav S.K., "On the Type-I optimality of nearly balanced incomplete block designs with small concurrence range", Statistica Sinica, 10, 1091-1116, 2000.

[14] Morgan J.P. and Uddin N., "Optimal, nonbinary, variance balanced desings", Statistica Sinica, 5, 535-546, 1995.

[15] Shan K.R. and Sinha B.K., Theory of Optimal Designs, Springer-Verlag, New York, 1989.

[16] Street D.J., "Optimality and efficiency: comparing block designs", Colbourn C.H and Dinitz J.H. (Eds.) The CRC Handbook of Combinatorial Designs, CRC Press, 561-564, 1996.

[17] Wallis W.D., "Regular graph designs", Journ. of Statist. Planning and Inference, 51, 273-281, 1996. 